\title{
Sofrimento moral e a dimensão ética no trabalho da enfermagem
}

\author{
Moral distress and the ethical dimension in nursing work \\ Sufrimiento moral y la dimensión de ética en el trabajo de enfermería
}

\section{Valéria Lerch Lunardi', Edison Luiz Devos Barlem', Michelle Salum Bulhosa', Silvana Sidney Costa Santos', Wilson Danilo Lunardi Filho', Rosemary Silva da Silveira', Ana Cristina Pretto Bao', Graziele de Lima Dalmolin'}

'Universidade Federal do Rio Grande. Núcleo de Estudos e Pesquisas em Enfermagem e Saúde. Rio Grande, RS

Submissão: 18/1 1/2008

Aprovação: 2 1/07/2009

\section{RESUMO}

Trabalhadores de enfermagem vivenciam problemas morais, dilemas morais e sofrimento moral, no seu cotidiano profissional. O sofrimento moral manifesta-se Quando os trabalhadores apresentam dificuldades para executar situações moralmente adeQuadas, segundo suas consciências. O texto tem como objetivo enfocar o sofrimento moral dos trabalhadores de enfermagem e sua relação com problemas morais e dilemas morais, abordando conceitos, vivências de sofrimento moral e possíveis estratégias a serem adotadas para o seu enfrentamento. Enfocar o sofrimento moral provocado pelo enfrentamento de problemas e dilemas morais no trabalho da enfermagem parece fundamental, não apenas para desvelar um fenômeno ainda pouco conhecido, mesmo que vivenciado Quase Que diariamente, mas, também, para destacar a necessidade de problematização e valorização da dimensão ética do trabalho em saúde.

Descritores: Enfermagem; Ética; Ética de enfermagem; Dano moral.

\section{ABSTRACT}

Nurses face moral problems, moral dilemmas and moral distress in their professional daily. The moral distress manifests itself when nurses demonstrate difficulties to face situations morally appropriate following their conscious. The study aims to focus about the nurses' moral distress and their relationship with moral problems and moral dilemmas presenting concepts, moral distress experiences and possible strategies to be adopted to face it. To focus on the moral distress provoked by facing the moral problems and dilemmas in nursery work seems to be primordial not just to reveal a phenomenon still a little known even experienced almost every day but, also to headline the need of problematization and valorization of the ethical dimension of work in health.

Descriptors: Nursing; Ethics; Ethics nursing; Moral damage.

\section{RESUMEN}

Trabajadores de enfermería vivencian problemas morales, dilemas morales y sufrimiento moral en su trabajo diario. Sufrimiento moral surge mientras los trabajadores de enfermería presentan dificultades para el desempeño de situaciones moralmente adecuadas según su conciencia. El texto tiene como objetivo centrarse sufrimiento moral de trabajadores de enfermería y su relación con problemas morales y dilemas morales, centrándose en conceptos, experiencias de sufrimiento moral y posibles estrategias a adoptar para su enfrentamiento. Direccionar sufrimiento moral causado por enfrentamiento de problemas y dilemas morales en el trabajo de enfermería, parece esencial no soló para develar un fenómeno todavía poco conocido, incluso experimentado casi a diario, pero también, poner de relieve la necesidad de problematización y recuperación de la dimensión ética del trabajo en salud.

Descriptores: Enfermería; Ética; Ética de enfermería; Daño moral. 


\section{INTRODUÇÃO}

Vivemos em um mundo em Que é facilmente percebida uma crise de valores. Nos hospitais, verificamos traços dessa desestruturação de valores, cuja dimensão parece assumir aspectos de normalidade, de uma nova cultura inserida nas várias maneiras de cuidar, nas posturas assumidas pelos trabalhadores ${ }^{(1)}$.

Em meio a essa situação, parece um paradoxo expressar o cuidado em sua forma mais sublime, tentando aliviar o sofrimento do outro, Quando o sofrimento inicial pode estar intrínseco no trabalho da própria enfermeira e sua equipe, sem Que seja percebido o real motivo. Várias manifestações de sofrimento, atreladas à constante necessidade de negação de valores pessoais, crenças e saberes são percebidas no mundo do trabalho, situações também relacionadas com o atendimento prestado por equipes Que agem com desrespeito e negação aos direitos dos próprios doentes como cidadãos $^{(2)}$.

Assim, trabalhadores da enfermagem vivenciam problemas, conflitos e dilemas morais, a partir de situações consideradas parte do seu cotidiano profissional, como a falta de consentimento informado de pacientes, antes de procedimentos diagnósticos e terapêuticos; a obstinação terapêutica; práticas profissionais Questionáveis e desigualdade na distribuição de recursos, em especial os destinados à equipe de enfermagem, o que lhes vem provocando sofrimento moral, sem Que esta temática seja suficientemente explorada $^{(3,4)}$.

O sofrimento moral dos trabalhadores de enfermagem, primariamente, decorre do fato de Que o trabalho da enfermagem é desenvolvido em um espaço social do sistema de cuidado em saúde, Que situa seus trabalhadores em uma grande proximidade dos pacientes, por períodos prolongados de tempo. Os trabalhadores de enfermagem e, em especial, as enfermeiras não são moralmente superiores, embora pareça mais fácil tomar decisões relacionadas ao corte de gastos e redução de pessoal, à distância, Quando não se mostra necessário visualizar as possíveis conseqüências destas tomadas de decisão, ou seja, "pode ser moralmente menos pesado dar ordens do Que executá-las ou conviver proximamente com as suas conseQüências" ${ }^{(5)}$.

Assim, com a elaboração desta reflexão tivemos como objetivo enfocar o sofrimento moral dos trabalhadores de enfermagem e sua relação com problemas morais e dilemas morais. Inicialmente, serão apresentados conceitos entendidos como fundamentais para o entendimento desta temática e, a seguir, possíveis estratégias a serem adotadas para o enfrentamento do sofrimento moral vivenciado pelos trabalhadores da enfermagem.

\section{ÉTICA, PROBLEMAS E DILEMAS MORAIS E SOFRIMENTO MORAL}

Ética é um termo alusivo a várias formas de analisar e entender a vida moral. A moral expressa um sistema de valores, emergindo normas reconhecidas como corretas em grupos sociais. A ética fundamenta-se em três pré-requisitos: percepção dos conflitos, autonomia e coerência frente aos conflitos ${ }^{(6)}$.

A enfermagem, historicamente, é uma profissão em Que as enfermeiras atuam como agentes morais, apropriando-se dos conceitos de compromisso, sensibilidade, autonomia, senso de dever, julgamento, conflito, competência e certeza ${ }^{(7)}$. O agenciamento moral refere-se ao desejo de prover cuidados de Qualidade, o Que é reconhecido tanto como uma necessidade, Quanto uma responsabilidade moral. Assim, a enfermeira torna-se agente moral do doente ${ }^{(8)}$

Problemas éticos vivenciados podem ser divididos em três categorias: incerteza moral, dilema moral e sofrimento moral. $\mathrm{Na}$ incerteza moral, o trabalhador reconhece uma situação inadeQuada ou incorreta, Questiona-se e apresenta sentimentos de tensão, frustração e incômodo, mas não percebe esta situação como parte de um problema ético ${ }^{(9)}$.

Dilemas morais são representados pela obrigação em escolher um determinado caminho entre dois ou mais cursos a seguir, porém, com uma única opção de escolha, sem Que nenhum deles seja mais correto do Que os outros ${ }^{(10)}$. Surgem, Quando a indecisão por uma ação recai sobre valores contraditórios ou cursos incompatíveis de ação. A característica fundamental dos dilemas morais é a indecisão no conflito(II).

Portanto, o dilema pode levar ao resíduo moral, Que são as marcas decorrentes do sofrimento experienciado, rompendo com a integridade moral, deixando traços na personalidade e nos valores construídos ao longo do tempo. A integridade moral refere-se à relação indissociável entre a integridade profissional e pessoal, dizendo respeito a um padrão moralmente aceito por nossa sociedade frente à ética. Os valores de uma pessoa são integrados à personalidade, tanto para o desenvolvimento pessoal, Quanto para alcançar a integridade moral, Que significa desenvolver uma perspectiva crítica através de um ponto de vista Que permita analisar, aceitar ou rejeitar situações novas. Quando a integridade moral é prejudicada, pode ocorrer sofrimento moral e, até mesmo, o abandono da profissão ${ }^{(10)}$.

Portanto, vivenciar situações de dilema moral pode provocar sofrimento moral, pois o trabalhador da enfermagem sabe o Que é correto e o Que deveria ser feito, mas não pode seguir o rumo de sua consciência, seja por obstáculos institucionais, falta de tempo, relutância das chefias, constrangimentos legais ou barreiras relacionadas ao poder médico, seja por dificuldades individuais de enfrentamento da situação ${ }^{(9,10)}$.

O sofrimento moral na prática da enfermagem foi descrito, inicialmente, como um desequilíbrio psicológico ocasionado por sentimentos dolorosos Que ocorrem Quando os trabalhadores de enfermagem não podem executar situações moralmente adeQuadas, segundo suas consciências ${ }^{(9)}$. Quando o sofrimento moral se faz presente, o impacto para o doente pode ser a falta de advocacia por parte da enfermagem e situações em Que os trabalhadores evitam o contato com os doentes, causando um aumento no desconforto e no próprio sofrimento do cliente frente à doença e ao tratamento ${ }^{(7)}$.

Esse sofrimento pode resultar em respostas no trabalhador como o descontentamento com o trabalho; possibilidade de aumento no número de doenças laborais; redução da carga de trabalho; afastamento, frieza nas relações, como recursos de defesa; visão do doente como uma atividade/trabalho e não como um ser humano; insônia, ansiedade, depressão e incapacidade de concentração; medo, cólera, trauma; mudanças de personalidade freeüentes, assim como sentimentos de culpa; cinismo nas convicções religiosas ou atitudes de maior fé; sentimentos de fraqueza, de falta de apoio moral e de falta de apoio profissional e solidão profissional ${ }^{(7,12)}$. 
No Que se refere à correlação entre a intensidade de sofrimento moral e a idade do trabalhador de enfermagem, foi constatada uma correlação negativa, o Que pode ser justificado pelo papel da experiência, no sentido de evitar problemas éticos. No entanto, essa correlação ainda é baixa, em níveis estatísticos ${ }^{(13)}$, o que também pode estar associado à carga de conhecimentos éticos associados à capacidade de assumir responsabilidades frente a um dilema moral $^{(14)}$. Assim, enfermeiras inexperientes enfrentam níveis mais altos de sofrimento moral, pela tensão e conflitos entre seus valores individuais e obstáculos externos, apresentando maior tendência em abandonar suas convicções sobre os direitos do doente, chegando, em alguns casos, ao erro ${ }^{(15)}$.

\section{TRABALHO DA ENFERMAGEM: VIVÊNCIAS DE SOFRIMENTO MORAL}

É notória a intensidade cada vez maior da vivência sistemática de dilemas morais e sofrimento moral por parte das enfermeiras, durante o processo de trabalho. Avanços da ciência têm permitido aos trabalhadores de saúde novas possibilidades de tratamento, incorporadas ao cuidado em saúde sem suficiente reflexão sobre seus riscos e benefícios e possíveis conseqüências sobre o prolongamento da vida e sua Qualidade ${ }^{(10)}$.

Os custos dessas alternativas tornam-se altos, mas escassos para a população. Podem constituir-se em fonte de sofrimento, servindo, assim, para o surgimento de problemas éticos e de conflitos Que testam incessantemente as virtudes morais das enfermeiras, já Que interferem no curso de vida dos doentes sob sua responsabilidade ${ }^{(16)}$.

Desse modo, a prescrição de cuidados fúteis, a solicitação de exames diagnósticos aparentemente desnecessários ${ }^{(1,17)}$; a falta de consentimento para a prescrição de condutas médicas ${ }^{(18)}$; o despreparo para o cuidado do outro no processo de morrer, agravado por situações de morte reconhecidas como evitáveis, em decorrência de negligência, poderão ser fonte de sofrimento moral aos trabalhadores $^{(4)}$. Qualitativamente, os sentimentos de raiva e tristeza são os mais citados na literatura como efeitos biopsicossociais ocasionados pelo sofrimento moral, sendo a introspecção a maior característica da equipe de enfermagem, Que pouco ou nenhum apoio recebe durante o enfrentamento de seus conflitos ${ }^{(17)}$.

As enfermeiras possuem mais responsabilidades do Que autoridade, no que diz respeito à jornada de trabalho. Possivelmente, o cumprimento autoritário das rotinas solicitadas pela equipe médica pode desequilibrar o senso de autoridade e de responsabilidade sobre o cuidado do doente ${ }^{(19)}$. Por outro lado, é esperado Que as enfermeiras aceitem a autoridade médica, sem participar no processo de tomada de decisão acerca dos cuidados dispensados aos doentes ${ }^{(20)}$. Todavia, suas relações mais próximas com os doentes, comparadas com o nível relacional de outros profissionais, podem contribuir para potencializar a ocorrência de um número maior de conflitos $^{(10)}$.

Frente a uma situação de sofrimento moral, duas alternativas apresentam-se para a enfermeira: a defesa do doente, com o possível desencadeamento de frustrações para o profissional, ou o enfrentamento de sentimentos de impotência, desmoralização, desamparo, desespero, raiva, aflição e culpa por fracasso frente ao doente $^{(18)}$. A percepção das enfermeiras sobre sua possibilidade de exercer poder na instituição, para a resolução de dilemas e Questões éticas ${ }^{(21-24)}$, parece fundamental para a sua decisão de uma tomada de ação ou não para este enfrentamento.

Mesmo Que conduzidas por práticas baseadas em códigos morais individuais, as pressões ambientais levam ao conformismo por parte das enfermeiras Que, gradualmente, se tornam mais distantes dos doentes $^{(25)}$. A enfermeira, ao afastar-se do doente e de seus cuidados, pode negar-lhe importantes benefícios, culminando em problemas de relacionamento na instituição, além de uma comunicação ineficaz com a equipe que lhe dispensa cuidados muito fragmentados.

Assim, a organização hospitalar aparece como o elemento de maior influência no cotidiano profissional, considerando-a como uma das grandes responsáveis pela crise de Qualidade no atendimento, destacando que os problemas éticos de enfermagem são sistemáticos e não fortuitos, tendo uma previsibilidade temporal, não sendo de responsabilidade das pessoas, mas do sistema. Os enfermeiros, geralmente, enfrentam problemas práticos morais sobre como realizar uma tarefa, frente à oposição de outras pessoas ou a regras estabelecidas, descaracterizando-se, dessa forma, tais vivências como um dilema ético. Dessa maneira, os trabalhadores de enfermagem geralmente não vivenciam dilemas morais, mas, sim, sofrimento moral(26).

A tensão é um fator Que tem influências negativas na percepção ética, provocando sentimentos Que levam à percepção do não cumprimento das obrigações morais ${ }^{(27)}$. Embora a tensão possa ser uma influência positiva motivadora de mudanças, o sofrimento moral desencadeia a ocorrência de respostas negativas no ambiente de trabalho ${ }^{(13)}$. Portanto, o desconhecimento acerca das Questões éticas e as tensões profissionais conduzem os trabalhadores ao sofrimento moral, com impactos negativos na Qualidade dos cuidados prestados $^{(28)}$

\section{O ENFRENTAMENTO DO SOFRIMENTO MORAL: POSSIBILIDADES E INCERTEZAS}

As soluções para a maior parte dos dilemas profissionais estão muito além do estudo da bioética, reQuerendo a modificação dos ambientes Que necessitam se tornar mais consistentes no Que tange à prática ética ${ }^{(10)}$. Uma estratégia para os problemas Que conduzem ao sofrimento moral poderá ser o desenvolvimento de programas de educação e ética direcionados aos trabalhadores da enfermagem, discutindo uma variedade de temas, de acordo com a necessidade da instituição e os componentes éticos envolvidos no cuidado aos doentes.

Esses programas deveriam abordar valores da profissão; identificação do social frente à moralidade privada; preservação da integridade moral; advocacia do doente; cuidado como função primordial da enfermagem; julgamento moral em enfermagem; poder das enfermeiras frente às decisões médicas ${ }^{(15)}$, pois a redução de mortalidade em alguns hospitais parece estar associada com a organização da enfermagem nestas instituições: status aumentado da enfermeira, autonomia, controle possibilitado às enfermeiras e o impacto resultante destes comportamentos em favor dos pacientes, superando uma Questão numérica de enfermeiras e de suas credenciais $^{(22)}$.

Para minimizar os efeitos do sofrimento moral, pode-se ainda promover discussões de temas relacionados à ética entre estudantes e trabalhadores de enfermagem, utilizando casos clínicos como forma 
de melhor introduzi-los nas Questões bioéticas. Ainda, sugere-se criar diretrizes de cuidado paliativo para as práticas profissionais, teoricamente minimizando o sofrimento moral através da adoção, por parte dos hospitais, de protocolos de decisão ética ${ }^{(17)}$.

Uma aproximação das equipes médica e de enfermagem requer a construção de uma comunidade moral nos ambientes hospitalares, por meio de um forte trabalho de equipe multiprofissional Que apóie a integridade moral dos envolvidos e suas decisões, discutindo os possíveis problemas. A verdade necessita ser sempre abordada como estratégia para fornecer melhores recursos para a decisão participativa entre equipe e familiares ${ }^{(10)}$.

Outra estratégia é a multidisciplinaridade, Que poderá ajudar a definir o caminho a seguir, reduzindo os impactos do sofrimento moral sobre o cuidado dos doentes ${ }^{(20,29)}$. A formação dos comitês de ética torna-se também uma importante estratégia. As instituições de saúde necessitam alargar a disponibilidade dos comitês de ética para discutir os problemas institucionais surgidos nas rotinas diárias e para apoiar os problemas Que, em grande parte, surgem em decorrência de fragilidades da formação profissional ${ }^{(13)}$.

\section{CONSIDERAÇÕES FINAIS}

Trabalhadores de enfermagem, cotidianamente, vivenciam problemas e conflitos morais Que podem lhes provocar sofrimento moral. Contraditoriamente, o sofrimento moral, apesar de doloroso, deveria constituir-se num importante mobilizador para o
Questionamento, o diálogo e o enfrentamento coletivo, na instituição, das inúmeras tensões de valores presentes nas ações profissionais, com repercussões positivas e negativas para o cuidado dos doentes. Vivenciar o sofrimento moral de modo solitário, isolado, sem interconexão com os demais trabalhadores, apenas intensifica o peso negativo deste sentimento, podendo contribuir para a sensação de impotência, desamparo e culpa com o afastamento dos doentes e de seu cuidado, bem como para o abandono da profissão.

Portanto, para o exercício saudável da enfermagem, são fundamentais: o diálogo aberto entre chefias e enfermeiras, no sentido de buscar medidas para evitar frustrações, prevendo mudanças; maior envolvimento dos enfermeiros no desenvolvimento de políticas organizacionais Que visem a nutrir melhores valores individuais; encorajamento para participar dos comitês de ética; presença de educação permanente Que fortaleça as escolhas éticas; clarificação contínua de valores, a partir de uma ego-reflexão ou de atividades grupais Que visem à manutenção de ideais individuais vivenciados, tendo como parâmetros o código de ética dos profissionais da enfermagem.

Enfocar o sofrimento moral provocado por problemas e dilemas morais presentes no trabalho da enfermagem, na assistência, no ensino e na pesQuisa, parece fundamental não apenas para desvelar um fenômeno por muitos ainda desconsiderado, apesar de vivenciado Quase que diariamente, mas, também, para destacar a necessidade de problematização e valorização da dimensão ética do trabalho em saúde.

\section{REFERÊNCIAS}

I. Bulhosa MS. Sofrimento moral no trabalho da enfermagem [dissertação]. Rio Grande: Programa de Pós-Graduação em Enfermagem, Universidade Federal do Rio Grande; 2006.

2. Selli L. Bioética na Enfermagem. São Leopoldo: Unisinos; 1999.

3. Carvalho KK. A obstinação terapêutica como uma Questão ética [dissertação]. Rio Grande: Programa de Pós-Graduação em Enfermagem, Universidade Federal do Rio Grande; 2005.

4. Dalmolin GL, Lunardi VL. O sofrimento moral para os trabalhadores de enfermagem no desempenho profissional: aproximações e distanciamentos de duas realidades [monografia]. Rio Grande: Curso de Enfermagem e Obstetrícia, Universidade Federal do Rio Grande; 2007.

5. Peter E, Liaschenko J. Perils of proximity: a spatiotemporal analysis of moral distress and moral ambiguity. Nurs InQuiry 2001; I I (4): 218-25.

6. Segre M, Cohen C, organizadores. Bioética. São Paulo: EDUSP; 2002.

7. Corley MC. Nurse moral distress: a proposed theory and research agenda. Nurs Ethics 2002; 9(6): 636-50.

8. O'Haire SE, Blackford IC. Nurses' moral agency in negotiating parental participation in care. Int I Nurs Pract 2005; I ( I): 250-6.

9. Jameton A. Nursing practice: the ethical issues. New Jersey: Prentice-Hall; 1984.

10. Hardingham LB. Integrity and moral residue: nurses as participants in a moral community. Nurs Philosophy 2004; 5(1): 127-34.

11. Jameton A. Dilems of moral distress: moral responsibility and nursing practice. Clinical Issues 1993;4(4):542-51.

12. Elpern EH, Covert B, Kleinpell R. Moral distress of staff nurses in a medical intensive care unit. Am J Crit Care 2005; 14(6): 523-30.

13. Corley MC, Minick P, Elswick RK, Jacobs M. Nurse moral distress and ethical work environment. Nurs Ethics 2005;12(4):38190.

14. Sporrong SK, Hölglund AT, Arnetz B. Measuring moral distress in pharmacy and clinical practice. Nurs Ethics 2006; 13(4): 416-27.

15. Ham K. Principled thinking: a comparison of nursing students and experienced nurses. I Contin Educ Nurs 2004; 35(2): 6673.

16. Kopala B, Burkhart L. Ethical dilemma and moral distress: proposed new NANDA diagnoses. Int I Nurs Terminol Classif 2005; 16(1): 3-13.

17. Gutierrez KM. Critical care nurses' perceptions of and responses to moral distress. Dimen Crit Care Nurs 2005;C24(5):C22941 .

18. Ferrel BR. Understanding moral distress of nurses witnessing medically futile care. Oncol Nurs Forum 2006; 33(5): $922-$ 30.

19. Corley MC, Elswick RK, Gorman M, Clot T. Development and evaluation of moral distress scale. I Adv Nurs 200 I; 33(2): 250-6.

20. Austin W, Lemermeyer G, Goldberg L, Bergum V. Moral distress 
in healthcare practice: the situation of nurses. HEC Forum 2005; 17(1): 33-48.

21 . Liashenko I. Feminist ethics and cultural ethos: revisiting a nursing debate. Adv Nurs Sci 1993; 15(4): 7 I-8I.

22. Aiken LH, Smith HL, Lake ET. Lower medicare mortality among a set of hospitals known for good nursing care. Med Care 1994; 32(8): 771-87.

23. Erlen IA, Frost B. Nurses' perceptions of powerlessness in influencing ethical decisions. West I Nurs Res 1991; 13(3): 397-407.

24. Penticuff $\mathrm{j}$, Walden $\mathrm{M}$. Influence of practice environment and nurse characteristics on perinatal nurses' responses to ethical dilemmas. Nurs Res 2000; 49(2): 64-72.
25. Kelly B. Preserving moral integrity: a follow-up study with new graduate nurses. I Adv Nurs 1998; 28(1): I I 34-45.

26. Chambliss DF. Beyond caring: hospital nurses, and the social organization of ethics. Chicago: The university of Chicago Press; 1996.

27. Severinsson E. Moral stress and burnout: Qualitative content analysis. Nurs Health Sci 2003; 5(1): 59-66.

28. Cohen-Mansfield J, Noelker L. Nursing staff satisfaction in longterm care: an over-view. In: Cohen-Mansfield I, Ejaz FK, Werner P, editors. Satisfaction surveys in long-term care. New York: Springer; 2000. p. 52-75.

29. McCaffree D. Moral distress and the intensive care unit. Crit Care Med 2006; 34(I2): 3049-50. 